\title{
An fMRI Study of Local Synchronization in Different Subfrequency Bands during the Continuous Feedback of Finger Force
}

\author{
Hang Zhang, ${ }^{1,2,3}$ Zhong-Zhan Gao, ${ }^{2,3}$ and Yu-Feng Zang ${ }^{1,2,3}$ \\ ${ }^{1}$ Paul C. Lauterbur Research Center for Biomedical Imaging, Shenzhen Institutes of Advanced Technology, \\ Chinese Academy of Sciences, Shenzhen 518055, China \\ ${ }^{2}$ Center for Cognition and Brain Disorders and the Affiliated Hospital, Hangzhou Normal University, Hangzhou 310015, China \\ ${ }^{3}$ Zhejiang Key Laboratory for Research in Assessment of Cognitive Impairments, Hangzhou 310015, China
}

Correspondence should be addressed to Hang Zhang; hang.zhang@siat.ac.cn

Received 1 August 2014; Accepted 4 January 2015

Academic Editor: Xi-Nian Zuo

Copyright (C) 2015 Hang Zhang et al. This is an open access article distributed under the Creative Commons Attribution License, which permits unrestricted use, distribution, and reproduction in any medium, provided the original work is properly cited.

\begin{abstract}
Conventional functional magnetic resonance imaging (fMRI) studies on motor feedback employ periodical blocked paradigm which does not allow frequency analysis of brain activity. Here, we carried out an fMRI study by using a continuous paradigm, that is, continuous ( $8 \mathrm{~min}$ ) feedback of finger force. Borrowing an analytic method widely used in resting-state fMRI studies, that is, regional homogeneity ( $\mathrm{ReHo}$ ), we compared the local synchronization in some subfrequency bands between real and sham feedback, and the subbands were defined as Slow-6 $(0.0-0.01 \mathrm{~Hz})$, Slow-5 $(0.01-0.027 \mathrm{~Hz})$, Slow-4 $(0.027-0.073 \mathrm{~Hz})$, Slow$3(0.073-0.198 \mathrm{~Hz})$, and Slow-2 $(0.198-0.25 \mathrm{~Hz})$. Our results revealed that the five subfrequency bands of brain activity contributed to the changes of ReHo between real and sham feedback differently, and, more importantly, the changes in basal ganglia were only manifested in Slow-6, implicating the fact that ReHo in ultraslow band may be associated with the functional significance of BG, that is, motor control. These findings provide novel insights into the neural substrate underlying motor feedback, and properties of the ultraslow band of local synchronization deserve more attention in future explorations.
\end{abstract}

\section{Introduction}

The motor feedback is a technique that enables participants to effectively regulate some kinetic parameters such as muscle force [1], speed [2], and gestures [3]. It exhibits benefits in improving some motor functions like the stand balance [4], finger force [5], and bimanual coordination [6] and also subserves the motor function rehabilitation for the patients with brain disorders of Parkinson disease [7], brain damage [8], chronic stroke [9], and so forth. These clinical values prompt more and more investigations on the neural substrates underlying the motor feedback.

Neuroimaging investigations intensively indicate that the motor feedback involves intricate brain activity. Results from functional magnetic resonance imagining mostly revealed that the motor cortices (e.g., precentral gyrus and postcentral gyrus) [10, 11], basal ganglia [12], and visual cortices [13, 14] exhibit functional prominence for varied experimental conditions of motor feedback, such as precision versus power force grip [10, 13], force magnitude [15], duration of maintained force [16], feedback frequency [17], and maturation of force control [18]. The involvement of these brain areas mainly came from the investigations on a block paradigm which is intermitted periodically (such as 30 s); however, the motor feedback in practice, for example, when driving a car, usually lasts for several minutes/hours. During such longlasting feedback, sustained attention also plays important roles in motor control $[19,20]$. Thus, Dong and his colleagues proposed a continuous performing paradigm for the fMRI investigation of the motor feedback and revealed the altered 
brain activity in the visual cortex and the areas of the default mode network, for example, posterior cingulate cortex, while comparing real and sham feedback conditions [21].

Recently, frequency-dependent characteristics of brain activity have been reported by more and more fMRI investigations [22-25]. Some separate frequency bands of brain activity such as Slow-6 $(0.0-0.01 \mathrm{~Hz})$ [22], Slow-5 (0.01$0.027 \mathrm{~Hz})$, Slow-4 (0.027-0.073 Hz), Slow-3 $(0.073-0.198 \mathrm{~Hz})$ $[23,24]$, and Slow-2 $(0.198-0.25 \mathrm{~Hz})[23]$ are generated with specific properties and physiological functions. The frequency-dependent analysis of brain activity exhibits clinical usefulness for quantification and detection of the functional pathological changes in brain disorders such as Parkinson's disease [25]. Although these brain disorders could be treated clinically with the motor feedback, the brain activity of motor feedback remains to be understood in different subfrequency bands.

Brain activity measured with the fMRI signal exhibits the local synchronization of the time courses of neighboring voxels, which could be assessed through the measurement of regional homogeneity ( $\mathrm{ReHo}$ ). Therefore, the present study aims to examine the local synchronization in the subfrequency bands of Slow-6, Slow-5, Slow-4, Slow-3, and Slow2 during motor feedback. Conventional block paradigm involves the periodical intermission that does not allow frequency-dependent analysis. Thus, we performed the fMRI experiment by employing a continuous paradigm, that is, continuous feedback of finger force. Then, the differences in $\mathrm{ReHo}$ between real and sham feedback conditions were investigated in the subfrequency bands.

\section{Materials and Methods}

2.1. Participants. Forty-three right-handed college students participated in the study $(22.7 \pm 1.6$ years, range $19-25 ; 23$ females). No participant had the histories of brain injury, neurological illness, or psychiatric disorders. Five subjects were excluded due to the malfunction of experimental equipment or excessive head motion (head motion was $>2 \mathrm{~mm}$ translation or $>2^{\circ}$ rotation in any direction), and, at last, data from 38 subjects (mean age, $22.3 \pm 1.6$ years; 19 females) were involved in the further analysis. All experiments conducted in this study were approved by the Institutional Review Board of the National Key Laboratory of Cognitive Neuroscience, Beijing Normal University. All of the subjects gave written informed before scanning.

2.2. Experimental Design. The experimental procedure has been reported in our recent study [21]. Each participant first underwent a scanning of resting state for adapting to the fMRI environment. Then, two scanning sessions, one for continuous real feedback and one for continuous sham feedback, were performed. Each session lasted for $8 \mathrm{~min}$, and the order of the two sessions was counterbalanced across all participants. In the session of real feedback, the participants gripped a pressure sensor between the right index finger and thumb. This sensor is one module of an MRI-compatible physiological multichannel analyzer (model MP150, BIOPAC Systems,
Inc., Goleta, CA). The sampling frequency was $250 \mathrm{~Hz}$ and the pressure sensitivity was $0.01 \mathrm{~cm} \mathrm{H}_{2} \mathrm{O}$. The pressure was recorded by a sensor via an airtight tube, and the force of pressure was synchronously fed back to the participant via a projector. At the same time, each participant was requested to continuously maintain the pinch force at $20 \mathrm{~cm} \mathrm{H}_{2} \mathrm{O}$ as far as possible according to the visual feedback. This target force was set in order to reduce the possibility of muscular fatigue for each subject [26]. In the session of sham feedback, participants were also asked to maintain the pinch force at $20 \mathrm{~cm} \mathrm{H}_{2} \mathrm{O}$ as far as possible, and the visual feedback they received came from the performance of another participant in the session of real feedback. The aim of this procedure was to minimize the difference in visual presentation between real and sham feedback sessions. Because sham feedback of pinch force could be easily detected by the subject, we informed participants of this fact in advance and requested them to watch the feedback while keeping their own performance unaffected. Before each session, the participants had a short training period.

2.3. Image Acquisition. Brain scans were performed at the MRI Center of Beijing Normal University using a 3.0T Siemens whole-body MRI scanner. A single-shot T2* weighted, gradient-echo EPI sequence was used for functional imaging acquisition with the following parameters: $\mathrm{TR} / \mathrm{TE} / \mathrm{flip}$ angle $=2000 \mathrm{~ms} / 30 \mathrm{~ms} / 90^{\circ}$, acquisition matrix $=$ $64 \times 64$, field of view $(\mathrm{FOV})=200 \times 200 \mathrm{~mm}^{2}$, and thickness $/$ gap $=3.5 / 0.7 \mathrm{~mm}$. Thirty-three axial slices parallel to the AC-PC line were obtained in an interleaved order to cover the entire cerebrum and cerebellum. Then a T1-weighted sagittal three-dimensional magnetization-prepared rapid gradientecho (MPRAGE) sequence was acquired (128 sagittal slices, thickness/gap $=1.33 / 0 \mathrm{~mm}$, in-plane resolution $=256 \times 192$, $\mathrm{TR}=2530 \mathrm{~ms}$, TE $=3.39 \mathrm{~ms}$, inversion time $=1100 \mathrm{~ms}$, flip angle $=7^{\circ}$, and FOV $=256 \times 256 \mathrm{~mm}^{2}$ ).

\subsection{Data Analysis}

2.4.1. Preprocessing. The preprocessing was carried out using the Data Processing Assistant for Resting-State fMRI (DPARSF) [27] which is based on the Statistical Parametric Mapping (SPM8) (http://www.fil.ion.ucl.ac.uk/spm/) and Resting-State fMRI Data Analysis Toolkit (REST) [28] (http:// www.restfmri.net/). For each subject, the first 10 time points of the functional data of real/sham feedback were discarded to allow for signal stabilization. These images were further corrected for intravolume acquisition time delay between slices and intervolume geometrical displacement due to head movement. Then, all images were normalized to the standard Montreal Neurological Institute (MNI) template (resampled into $3 \times 3 \times 3 \mathrm{~mm}^{3}$ ) via parameters of individual structural image spatial normalization based on unified segmentation [29]. Six head motion parameters (three rigid body translations and three rotations) were regressed out from the fMRI data, and the linear trends were removed from the time courses of the voxels in each image. According to previous 
TABLE 1: Clusters showing significant main effect for the feedback condition. The statistical threshold was set at $P<0.005$, cluster size $>98$.

\begin{tabular}{|c|c|c|c|c|c|c|}
\hline \multirow{2}{*}{ Brain regions } & \multirow{2}{*}{$\mathrm{L} / \mathrm{R}$} & \multirow{2}{*}{ BA } & \multicolumn{4}{|c|}{ Peak MNI coordinates } \\
\hline & & & $x$ & $y$ & $z$ & $F(1,370)$ \\
\hline Inferior/middle occipital Gyrus/calcarine & $\mathrm{L} / \mathrm{R}$ & $17 / 18$ & 12 & -90 & 0 & 78.86 \\
\hline PCC & $\mathrm{L} / \mathrm{R}$ & 31 & 0 & -45 & 33 & 28.31 \\
\hline $\mathrm{mPFC}$ & $\mathrm{L} / \mathrm{R}$ & 9 & -6 & 48 & 27 & 18.36 \\
\hline BG & $\mathrm{L}$ & & -24 & -12 & 12 & 13.39 \\
\hline
\end{tabular}

investigations [22-25], we used band-pass filtering to subdivide the whole detectable frequency range $(0-0.25 \mathrm{~Hz})$ into five subfrequency bands, namely, Slow- $6(0.0-0.01 \mathrm{~Hz})$, Slow$5(0.01-0.027 \mathrm{~Hz})$, Slow-4 (0.027-0.073 Hz), Slow-3 $(0.073-$ $0.198 \mathrm{~Hz})$, and Slow-2 $(0.198-0.25 \mathrm{~Hz})$. Then, for each subfrequency band, the filtered functional data were further assessed through a voxelwise measurement of the regional homogeneity (ReHo).

2.4.2. Regional Homogeneity (ReHo) Analysis. ReHo is an analytic method widely used in resting-state fMRI studies. It is a voxelwise measure of the brain activity by examining the synchronization of the time courses of a certain voxel and its adjacent neighboring voxels [30].

The ReHo analysis employs Kendall's coefficient of concordance (KCC) to measure the local synchronization of the time courses of neighboring voxels as follows [30]:

$$
W=\frac{\sum\left(R_{i}\right)^{2}-n(\bar{R})^{2}}{(1 / 12) K^{2}\left(n^{3}-n\right)},
$$

where $W$ is the KCC among given voxels, ranged from 0 to 1 ; $R_{i}$ is the sum rank of the $i$ th time point; $\bar{R}=((n+1) K / 2)$ is the mean of the $R_{i}$ 's; $K$ is the number of time courses within a measured cluster (here, $K=27$, one given voxel plus the number of its neighbors); $n$ is the number of ranks. The KCC was calculated for each 27 nearest neighboring voxels in a voxelwise manner and the KCC value was assigned to the central voxel of each 27-voxel cluster. For each subfrequency band, the ReHo analysis was conducted using DPARSFA. Individual ReHo image during real/sham feedback was generated within a whole-brain mask and nonbrain areas are excluded. The whole-brain mask was provided in REST [28]. The individual ReHo image for each frequency band during $\mathrm{real} / \mathrm{sham}$ feedback was then smoothed with a $6 \times 6 \times 6 \mathrm{~mm}$ full-width-at-half-maximum (FWHM) Gaussian kernel. Then, a two-way repeated measures analysis of variance (ANOVA) was performed with factors of the feedback condition ( 2 levels, real and sham) and the frequency band (5 levels, i.e., Slow-6, Slow-5, Slow-4, Slow-3, and Slow-2). Then, the resultant F-maps were corrected for multiple comparisons with the threshold of $P<0.005$ and cluster size $>98$ voxels, corresponding to a corrected $P$ value of $<0.05$ as determined by AlphaSim (http://afni.nimh.nih.gov/pub/dist/doc/manual/AlphaSim.pdf). For clusters showing significance in the main effect of the feedback condition factor, region of interest (ROI) was defined with a sphere of $6 \mathrm{~mm}$ radius which was centered at the peak coordinate. Then, for each ROI, the mean ReHo across all subfrequency bands and subjects was calculated for real and sham feedback conditions, respectively. For clusters showing significant interaction effect between factors of the feedback condition and the frequency band, we also defined ROIs with a sphere of $6 \mathrm{~mm}$ radius which was centered at the peak coordinate. ReHo of each ROI was extracted based on the frequency band and the feedback condition of every subject. Then, for each ROI, paired $t$-tests were further performed to examine the difference of ReHo between real and sham feedback in each subfrequency band. The tested results were corrected for multiple comparisons to a significant level of $P<0.05$ (Bonferroni correction across the five frequency bands).

\section{Result}

According to the main effect of the feedback condition factor, differences of ReHo between real and sham feedback were distributed in four clusters, including bilateral visual cortex (containing bilateral inferior occipital gyrus, bilateral middle occipital gyrus, and bilateral calcarine), bilateral posterior cingulate cortex (PCC), bilateral medial prefrontal cortex (mPFC), and left BG (mainly located in putamen) (Table 1 and Figure 1(a)). For these clusters, the mean ReHo across all investigated subfrequency bands and subjects were shown in Figures 1(b)-1(e). Visual cortex showed lower ReHo while comparing real feedback with sham feedback (Figure 1(b)). As Figure 1(c) shows, ReHo for the mPFC was greater in real feedback than it was in sham feedback. As to the PCC, real feedback recruited greater ReHo than sham feedback (Figure 1(d)), and, for the left BG, greater ReHo was observed in real feedback as compared with that in sham feedback (Figure 1(e)). The main effect of the frequency band factor was similar to the findings of the previous study [23], and it was not presented here because it is not the focus of the current study.

The interaction effect between factors of the feedback condition and the frequency band was observed in three clusters, that is, the bilateral PCC and both of the left and right basal ganglia (BG) (mainly containing putamen and caudate) (Figure 2(a) and Table 2). For the PCC, real feedback exhibited greater ReHo in the Slow-5 $(t(37)=3.71, P<0.005)$ and Slow-4 $(t(37)=3.75, P<0.005)$ than sham feedback (Figure 2(d)). As Figures 2(b) and 2(c) show, real feedback recruited greater ReHo in the left and right BG than sham feedback and these significant differences were only manifested in Slow-6 $(t(37)=4.38, P<0.005$ for the left BG and $t(37)=4.29, P<0.005$ for the right BG). 


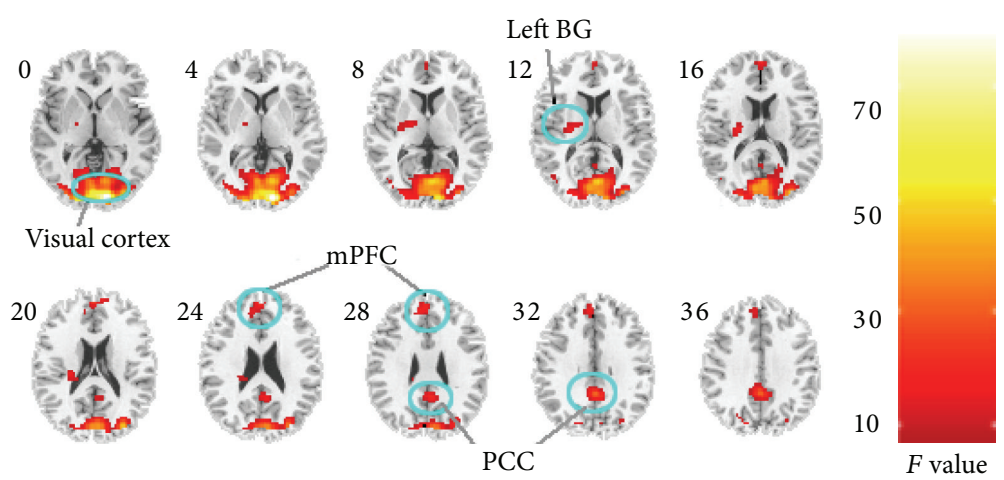

(a)

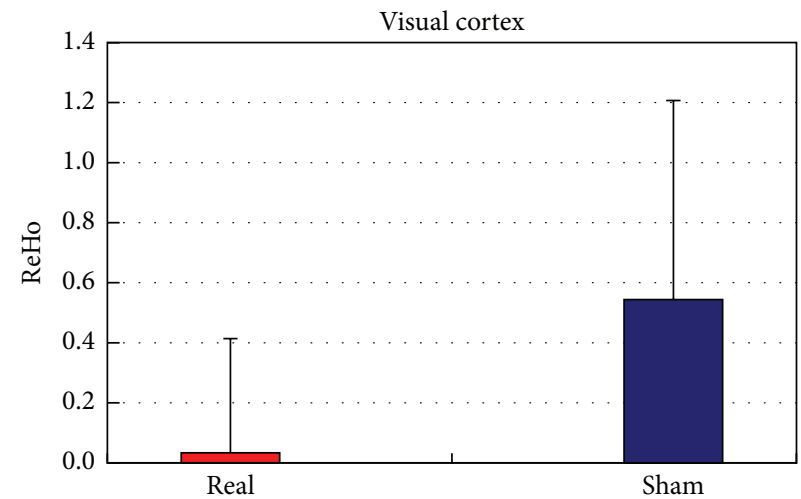

(b)

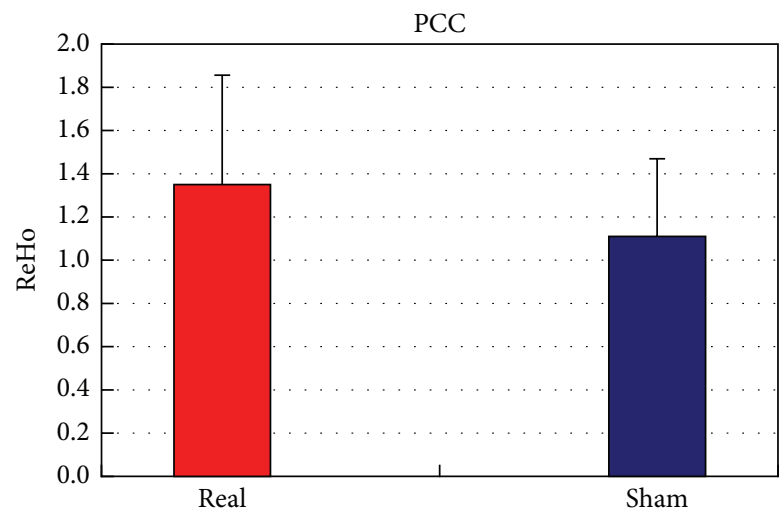

(d)

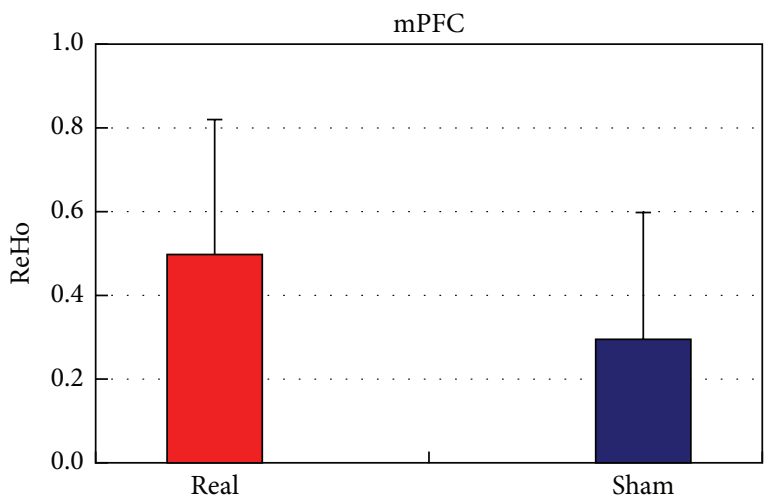

(c)

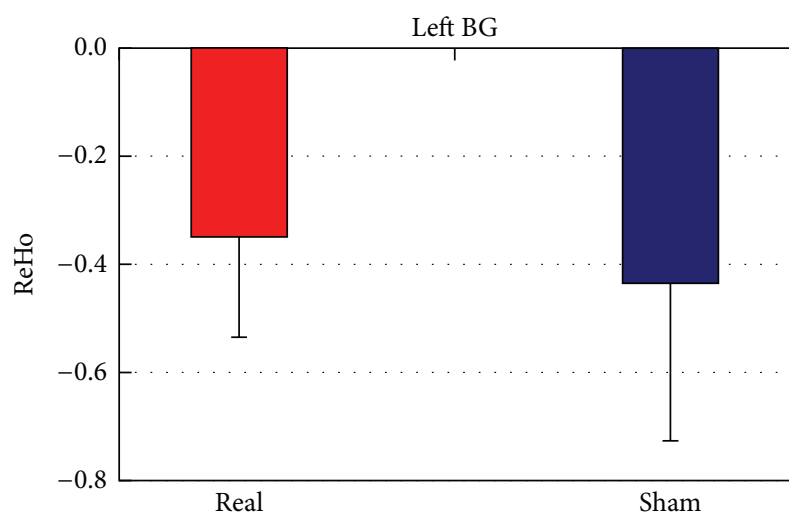

(e)

Figure 1: Clusters showing significant main effect of the feedback condition and ReHo of each cluster in all subfrequency bands for real/sham feedback. (a) Slice views of the spatial maps for the main effect of the feedback condition. (b) ReHo of the visual cortex in all subfrequency bands for real/sham feedback; (c) ReHo of the mPFC in all subfrequency bands for real/sham feedback; (d) ReHo of the PCC in all subfrequency bands for real/sham feedback; (e) ReHo of the left BG in all subfrequency bands for real/sham feedback. Red represents real feedback and blue represents sham feedback.

TABLE 2: Clusters showing significant interaction effect between the feedback condition and the frequency band. The statistical threshold was set at $P<0.005$, cluster size $>98$.

\begin{tabular}{|c|c|c|c|c|c|c|}
\hline \multirow{2}{*}{ Brain regions } & \multirow{2}{*}{$\mathrm{L} / \mathrm{R}$} & \multirow{2}{*}{ BA } & \multicolumn{4}{|c|}{ Peak MNI coordinates } \\
\hline & & & $x$ & $y$ & $z$ & $F(4,370)$ \\
\hline PCC & $\mathrm{L} / \mathrm{R}$ & $7 / 31$ & 0 & -66 & 33 & 10.08 \\
\hline $\mathrm{BG}$ & $\mathrm{L}$ & & -30 & 3 & 0 & 7.32 \\
\hline BG & $\mathrm{R}$ & & 33 & 3 & 6 & 7.79 \\
\hline
\end{tabular}




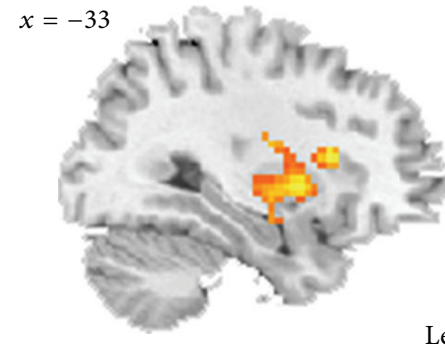

Left BG

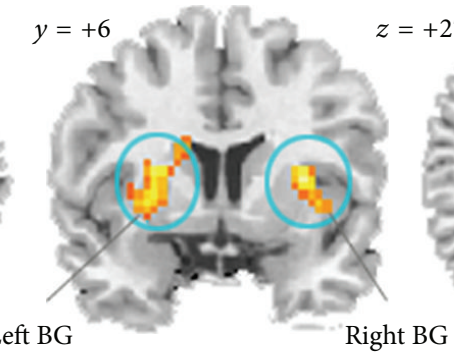

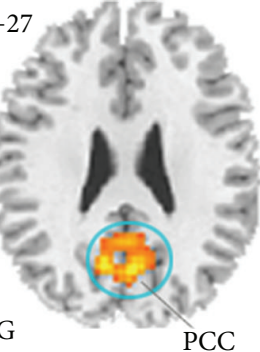

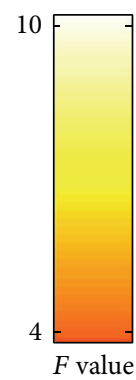

(a)
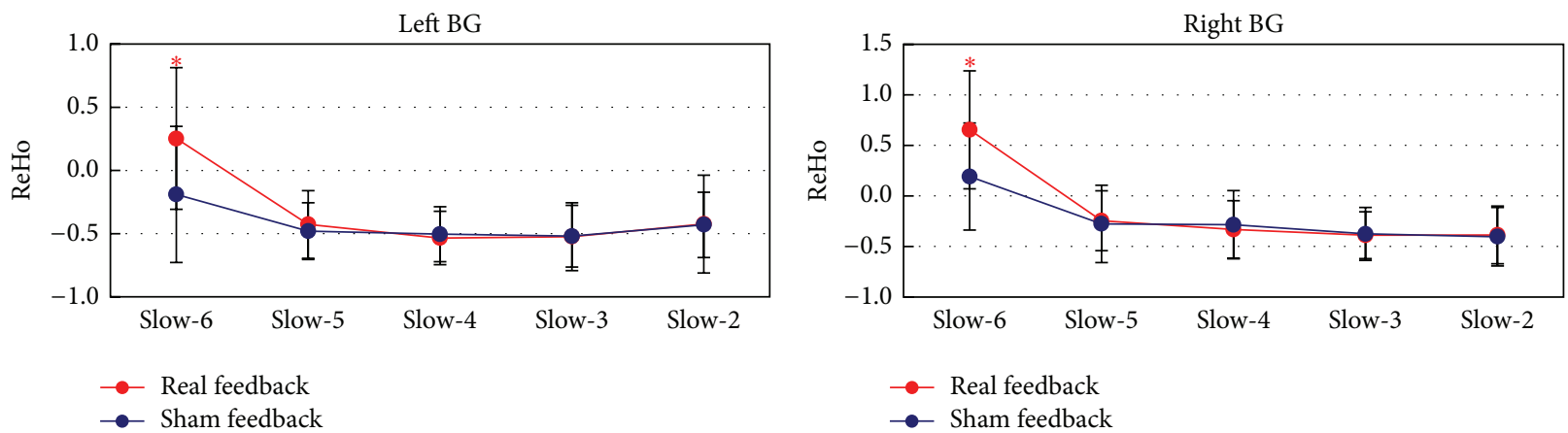

(b)

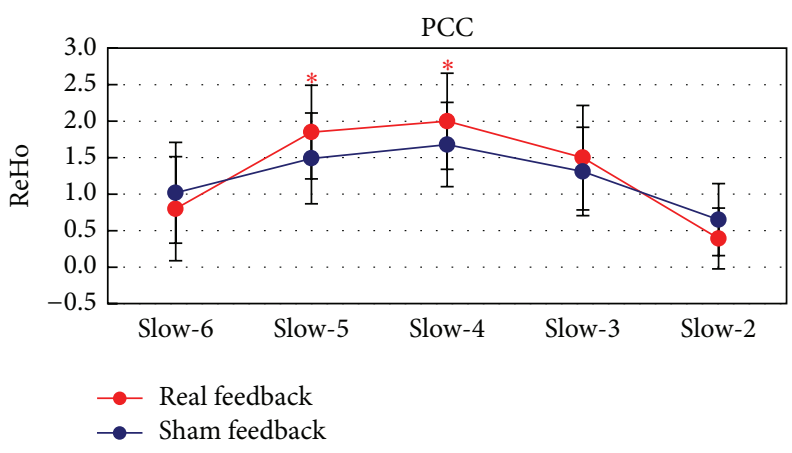

(d)

FIGURE 2: Clusters showing significant interaction effect between factors of the feedback condition and the frequency band and the relevant comparison results of ReHo in different frequency bands between real and sham feedback conditions. (a) Coronal, sagittal, and axial views of the spatial maps for the interaction effect between the feedback condition and the frequency band; (b) changes in ReHo of the left BG across the frequency bands during real and sham feedback; (c) changes in ReHo of the right BG across the frequency bands during real and sham feedback; (d) changes in ReHo of the PCC across the frequency bands during real and sham feedback. Red represents real feedback and blue represents sham feedback. * indicates the significant difference of ReHo between real and sham feedback. The statistical threshold was set at $P<0.05$, corrected for multiple comparisons.

\section{Discussion}

The present fMRI study investigated the neural substrate of motor feedback using a frequency-dependent analysis. The local synchronization of brain activity was assessed through a voxelwise measurement of ReHo in five separate subfrequency bands ranged from Slow-6 $(0.0-0.01 \mathrm{~Hz})$ to Slow$2(0.198-0.25 \mathrm{~Hz})$. Two intriguing results were observed: (1) as compared with sham feedback, real feedback recruited greater ReHo of the PCC, in Slow-5 and Slow-4; (2) ReHo differences in the left and right BG were mainly manifested in the ultraslow frequency band of Slow-6 which is less concerned in previous neuroimaging explorations.
Few previous investigations have performed fMRI investigations on the neural substrate of motor feedback in different subfrequency bands. This is probably because these investigations mostly employ the periodically blocked paradigm that is not suitable for the frequency-dependent analysis. The present study showed the benefits of the continuous paradigm and frequency-dependent ReHo analysis for examining the frequency-dependent fMRI signal characteristics in the process of task performing. The ReHo differences for the visual cortex and the mPFC were manifested in all but not some specific subfrequency bands. Real feedback recruited lower ReHo in visual cortex than sham feedback. The involvement of the visual cortex is mostly manifested in visually guided 
motor feedback $[13,14]$. It is thought that visual cortex mainly responds to update the visual information and further process the information for the adjustment of the force $[13,21,31]$. In our experiment, participants were requested to maintain their finger force according to real feedback, and, then, the visual processing may be more involved in this condition. Greater ReHo for the mPFC was observed while comparing real feedback with sham feedback. The mPFC is known to be highly sensitive to the sustained attention [32]. Thus, the greater ReHo in real feedback may be linked with the attentional processing of the visual stimuli [33].

The changes of ReHo in the PCC mainly came from Slow-5 and Slow- 4 when comparing real feedback with sham feedback. The Slow-5 and Slow-4 cover the frequency range of $0.01-0.073 \mathrm{~Hz}$ which is roughly equivalent to the typical low frequency band $(0.01-0.08 \mathrm{~Hz})[34,35]$. Mostly recruited in the default mode network in the typical low frequency band, the PCC has been identified as the hubs of this network chiefly responsible for attentional lapses and mind wandering $[36,37]$. In task state, these areas may play a role as a source of internal interference or noise and were suppressed as deactivation $[37,38]$, and the deactivation may further induce the elevation of ReHo. Thus, the greater ReHo in the PCC suggested that real feedback requires more suppression of internal interference than sham feedback, and, more importantly, our results indicated that the suppression was potentially associated with the local synchronization of the PCC in Slow-5 and Slow-4.

We observed that ReHo of the left and right BG in Slow6 is greater for real feedback than it is for sham feedback. Slow-6, as an ultraslow frequency band, is less concerned in previous neuroimaging explorations. In resting state, it was thought that this ultraslow frequency band may reflect very low frequency drift [39]. However, a recent study provides new insights into this issue by showing that oscillations lower than $0.02 \mathrm{~Hz}$ contribute more to ReHo in putamen during resting state [40]. Our results support this finding and further indicate that, during motor feedback, the oscillation in the ultraslow frequency band of Slow-6 is critical for ReHo in BG (including bilateral putamen and caudate). The BG is an important brain area for motor feedback. It is suggested that the $\mathrm{BG}$ is involved in the planning and parameterizing of motor control [41]. Thus, the ultraslow frequency band of brain oscillation during motor feedback may be associated with these functional roles of BG. Remarkably, the BG disorders such as Parkinson's disease mostly result in the decreased ReHo in the BG [42], and the motor feedback has been employed in the treatment of these disorders exhibiting therapeutic effectiveness [43]. Thus, the ultraslow frequency band of local synchronization during motor feedback may possess the therapeutic value in these clinical practices.

Nevertheless, the current study has some limitations. The sampling rate in the present study (2s) prevents us from performing the analysis in higher frequency band and we believed that fast sampling should provide more novel findings for motor feedback fMRI studies. Moreover, the results of the present study are restricted to the visual feedback, and the feedback presented in the auditory and sensory forms is commonly employed in practice. Further experimentation and investigation are still required to fully clarify these issues.

\section{Conclusion}

The present fMRI study shed light on the neural substrate of motor feedback by studying the local synchronization in the subfrequency bands ranged from Slow- 6 to Slow-2. Using the measurement of ReHo, we found that the five subfrequency bands exhibit distinct contributions to the changes of ReHo between real and sham feedback, which provided novel insights into the neural substrate of motor feedback. The result that changes in left and right BG mainly depended on the ultraslow frequency band of Slow-6, which potentially helps to understand properties of the ultraslow frequency band of local synchronization.

\section{Conflict of Interests}

The authors declare that there is no conflict of interests regarding the publication of this paper.

\section{Acknowledgments}

This work is supported by grants from the National Natural Science Foundation of China (81401481, 81271652, 81020108022, and 31471084), China Postdoctoral Science Foundation (2014M562225), and 973 Program (2015CB755500). Dr. Zang is partly supported by "Qian Jiang Distinguished Professor" Program. The authors thank Mr. Zhang-Ye Dong and Mr. Zhen-Xiang Zang for their data collection.

\section{References}

[1] J. W. Noble, J. J. Eng, and L. A. Boyd, "Effect of visual feedback on brain activation during motor tasks: an fMRI study," Motor Control, vol. 17, no. 3, pp. 298-312, 2013.

[2] D. D. Damian, A. H. Arita, H. Martinez, and R. Pfeifer, "Slip speed feedback for grip force control," IEEE Transactions on Biomedical Engineering, vol. 59, no. 8, pp. 2200-2210, 2012.

[3] J. A. Barrios, K. M. Crossley, and I. S. Davis, "Gait retraining to reduce the knee adduction moment through real-time visual feedback of dynamic knee alignment," Journal of Biomechanics, vol. 43, no. 11, pp. 2208-2213, 2010.

[4] R. Barclay-Goddard, T. Stevenson, W. Poluha, M. E. Moffatt, and S. P. Taback, "Force platform feedback for standing balance training after stroke," Cochrane Database of Systematic Reviews, vol. 18, no. 4, Article ID CD004129, 2004.

[5] N. J. Seo, H. W. Fischer, R. A. Bogey, W. Z. Rymer, and D. G. Kamper, "Use of visual force feedback to improve digit force direction during pinch grip in persons with stroke: a Pilot Study," Archives of Physical Medicine and Rehabilitation, vol. 92, no. 1, pp. 24-30, 2011.

[6] N. Lodha, C. Patten, S. A. Coombes, and J. H. Cauraugh, "Bimanual force control strategies in chronic stroke: finger extension versus power grip," Neuropsychologia, vol. 50, no. 11, pp. 2536-2545, 2012.

[7] D. E. Vaillancourt, A. B. Slifkin, and K. M. Newell, "Intermittency in the visual control of force in Parkinson's disease," Experimental Brain Research, vol. 138, no. 1, pp. 118-127, 2001. 
[8] G. Kriz, J. Hermsdörfer, C. Marquardt, and N. Mai, "Feedbackbased training of grip force control in patients with brain damage," Archives of Physical Medicine and Rehabilitation, vol. 76, no. 7, pp. 653-659, 1995.

[9] S. K. Naik, C. Patten, N. Lodha, S. A. Coombes, and J. H. Cauraugh, "Force control deficits in chronic stroke: grip formation and release phases," Experimental Brain Research, vol. 211, no. 1, pp. 1-15, 2011.

[10] H. H. Ehrsson, A. Fagergren, T. Jonsson, G. Westling, R. S. Johansson, and H. Forssberg, "Cortical activity in precisionversus power-grip tasks: an fMRI study," Journal of Neurophysiology, vol. 83, no. 1, pp. 528-536, 2000.

[11] S. Haller, D. Chapuis, R. Gassert, E. Burdet, and M. Klarhöfer, "Supplementary motor area and anterior intraparietal area integrate fine-graded timing and force control during precision grip," European Journal of Neuroscience, vol. 30, no. 12, pp. 24012406, 2009.

[12] S. T. Grafton and E. Tunik, "Human basal ganglia and the dynamic control of force during on-line corrections," The Journal of Neuroscience, vol. 31, no. 5, pp. 1600-1605, 2011.

[13] J. P. Kuhtz-Buschbeck, R. Gilster, S. Wolff, S. Ulmer, H. Siebner, and O. Jansen, "Brain activity is similar during precision and power gripping with light force: an fMRI study," NeuroImage, vol. 40, no. 4, pp. 1469-1481, 2008.

[14] S. A. Coombes, D. M. Corcos, L. Sprute, and D. E. Vaillancourt, "Selective regions of the visuomotor system are related to gaininduced changes in force error," Journal of Neurophysiology, vol. 103, no. 4, pp. 2114-2123, 2010.

[15] H. H. Ehrsson, A. Fagergren, and H. Forssberg, "Differential fronto-parietal activation depending on force used in a precision grip task: an fMRI study," Journal of Neurophysiology, vol. 85, no. 6, pp. 2613-2623, 2001.

[16] B. Keisker, M.-C. Hepp-Reymond, A. Blickenstorfer, and S. S. Kollias, "Differential representation of dynamic and static power grip force in the sensorimotor network," European Journal of Neuroscience, vol. 31, no. 8, pp. 1483-1491, 2010.

[17] S. A. Coombes, D. M. Corcos, and D. E. Vaillancourt, "Spatiotemporal tuning of brain activity and force performance," NeuroImage, vol. 54, no. 3, pp. 2226-2236, 2011.

[18] P. Halder, S. Brem, K. Bucher et al., "Electrophysiological and hemodynamic evidence for late maturation of hand power grip and force control under visual feedback," Human Brain Mapping, vol. 28, no. 1, pp. 69-84, 2007.

[19] B. S. Oken, M. C. Salinsky, and S. M. Elsas, "Vigilance, alertness, or sustained attention: physiological basis and measurement," Clinical Neurophysiology, vol. 117, no. 9, pp. 1885-1901, 2006.

[20] W. S. Helton, "Impulsive responding and the sustained attention to response task," Journal of Clinical and Experimental Neuropsychology, vol. 31, no. 1, pp. 39-47, 2009.

[21] Z.-Y. Dong, D.-Q. Liu, J. Wang et al., "Low-frequency fluctuation in continuous real-time feedback of finger force: a new paradigm for sustained attention," Neuroscience Bulletin, vol. 28, no. 4, pp. 456-467, 2012.

[22] Y. T. Lv, D. S. Margulies, A. Villringer, and Y. F. Zang, "Effects of finger tapping frequency on regional homogeneity of sensorimotor cortex," PLoS ONE, vol. 8, no. 5, Article ID e64115, 2013.

[23] X.-N. Zuo, A. di Martino, C. Kelly et al., "The oscillating brain: complex and reliable," NeuroImage, vol. 49, no. 2, pp. 1432-1445, 2010.

[24] Y. Han, J. Wang, Z. Zhao et al., "Frequency-dependent changes in the amplitude of low-frequency fluctuations in amnestic mild cognitive impairment: a resting-state fMRI study," NeuroImage, vol. 55, no. 1, pp. 287-295, 2011.

[25] Y. Hou, X. Wu, M. Hallett, P. Chan, and T. Wu, "Frequencydependent neural activity in Parkinson's disease," Human Brain Mapping, vol. 35, no. 12, pp. 5815-5833, 2014.

[26] H. van Duinen, R. Renken, N. Maurits, and I. Zijdewind, "Effects of motor fatigue on human brain activity, an fMRI study," NeuroImage, vol. 35, no. 4, pp. 1438-1449, 2007.

[27] C.-G. Yan and Y.-F. Zang, "DPARSF: a MATLAB toolbox for 'pipeline' data analysis of resting-state fMRI," Frontiers in Systems Neuroscience, vol. 4, article 13, 2010.

[28] X.-W. Song, Z.-Y. Dong, X.-Y. Long et al., "REST: a toolkit for resting-state functional magnetic resonance imaging data processing," PLoS ONE, vol. 6, no. 9, Article ID e25031, 2011.

[29] J. Ashburner and K. J. Friston, "Unified segmentation," NeuroImage, vol. 26, no. 3, pp. 839-851, 2005.

[30] Y.Zang, T. Jiang, Y. Lu, Y. He, and L. Tian, "Regional homogeneity approach to fMRI data analysis," NeuroImage, vol. 22, no. 1, pp. 394-400, 2004.

[31] D. E. Vaillancourt, K. R. Thulborn, and D. M. Corcos, "Neural basis for the processes that underlie visually guided and internally guided force control in humans," Journal of Neurophysiology, vol. 90, no. 5, pp. 3330-3340, 2003.

[32] M. Sarter, B. Givens, and J. P. Bruno, "The cognitive neuroscience of sustained attention: where top-down meets bottomup," Brain Research Reviews, vol. 35, no. 2, pp. 146-160, 2001.

[33] T. M. Gill, M. Sarter, and B. Givens, "Sustained visual attention performance-associated prefrontal neuronal activity: evidence for cholinergic modulation," The Journal of Neuroscience, vol. 20, no. 12, pp. 4745-4757, 2000.

[34] B. Biswal, F. Z. Yetkin, V. M. Haughton, and J. S. Hyde, "Functional connectivity in the motor cortex of resting human brain using echo-planar mri," Magnetic Resonance in Medicine, vol. 34, no. 4, pp. 537-541, 1995.

[35] M. J. Lowe, B. J. Mock, and J. A. Sorenson, "Functional connectivity in single and multislice echoplanar imaging using restingstate fluctuations," NeuroImage, vol. 7, no. 2, pp. 119-132, 1998.

[36] M. F. Mason, M. I. Norton, J. D. van Horn, D. M. Wegner, S. T. Grafton, and C. N. Macrae, "Wandering minds: the default network and stimulus-independent thought," Science, vol. 315, no. 5810, pp. 393-395, 2007.

[37] A. Anticevic, M. W. Cole, J. D. Murray, P. R. Corlett, X.-J. Wang, and J. H. Krystal, "The role of default network deactivation in cognition and disease," Trends in Cognitive Sciences, vol. 16, no. 12, pp. 584-592, 2012.

[38] M. D. Greicius and V. Menon, "Default-mode activity during a passive sensory task: uncoupled from deactivation but impacting activation," Journal of Cognitive Neuroscience, vol. 16, no. 9, pp. 1484-1492, 2004.

[39] T. Starck, J. Nissilä, A. Aunio et al., "Stimulating brain tissue with light-resting state fMRI analysis," in Proceedings of the International Society for Magnetic Resonance in Medicine, p. $1594,2011$.

[40] X. Song, Y. Zhang, and Y. Liu, "Frequency specificity of regional homogeneity in the resting-state human brain," PLOS ONE, vol. 9, no. 1, Article ID e86818, 2014.

[41] J. Prodoehl, D. M. Corcos, and D. E. Vaillancourt, "Basal ganglia mechanisms underlying precision grip force control," Neuroscience and Biobehavioral Reviews, vol. 33, no. 6, pp. 900908, 2009. 
[42] T. Wu, X. Long, Y. Zang et al., "Regional homogeneity changes in patients with Parkinson's disease," Human Brain Mapping, vol. 30, no. 5, pp. 1502-1510, 2009.

[43] D. E. Vaillancourt, A. B. Slifkin, and K. M. Newell, "Intermittency in the visual control of force in Parkinson's disease," Experimental Brain Research, vol. 138, no. 1, pp. 118-127, 2001. 

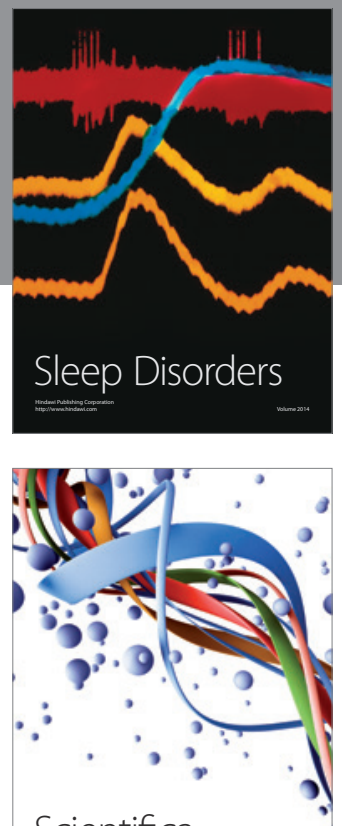

Scientifica
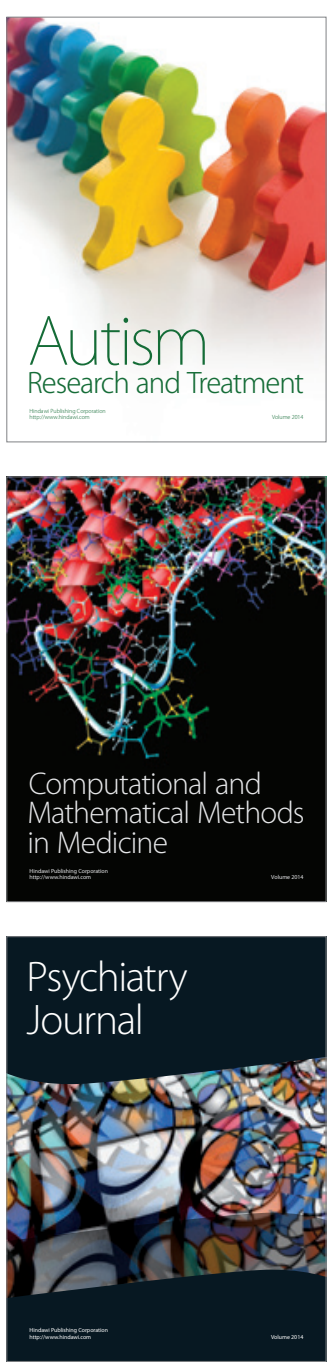
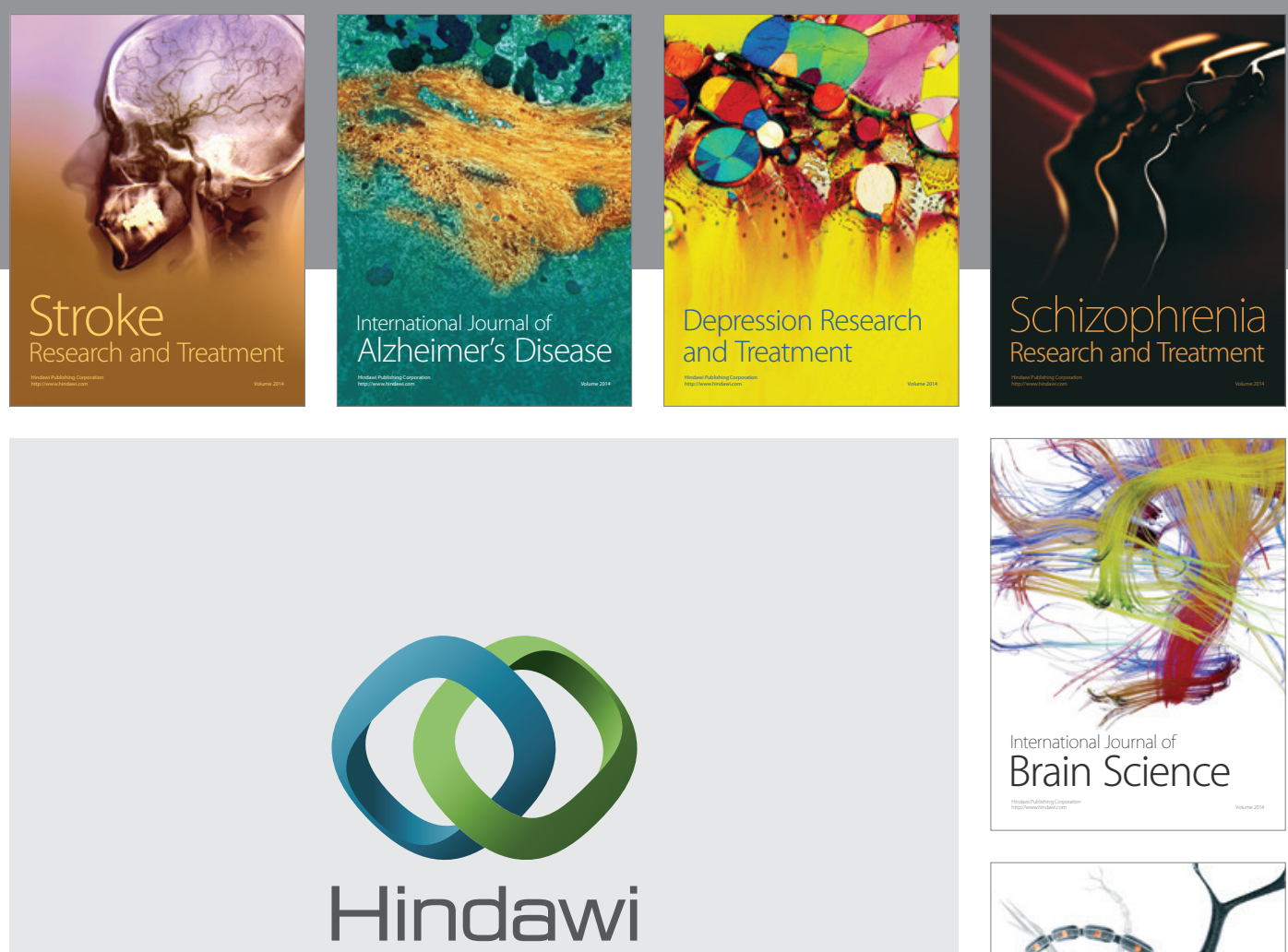

Submit your manuscripts at

http://www.hindawi.com
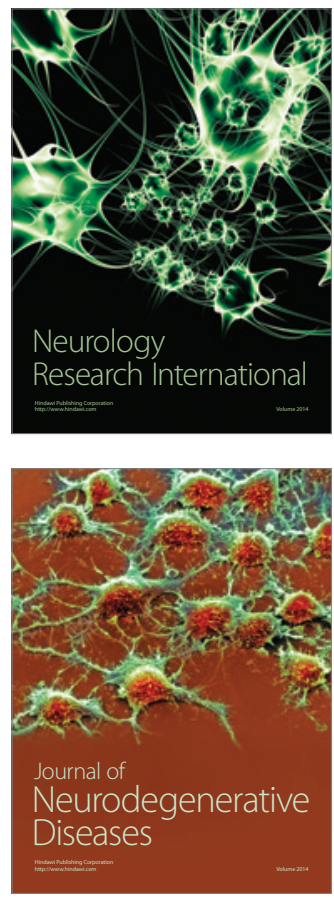

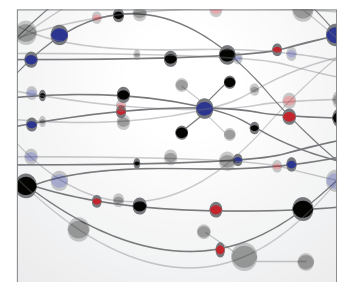

The Scientific World Journal
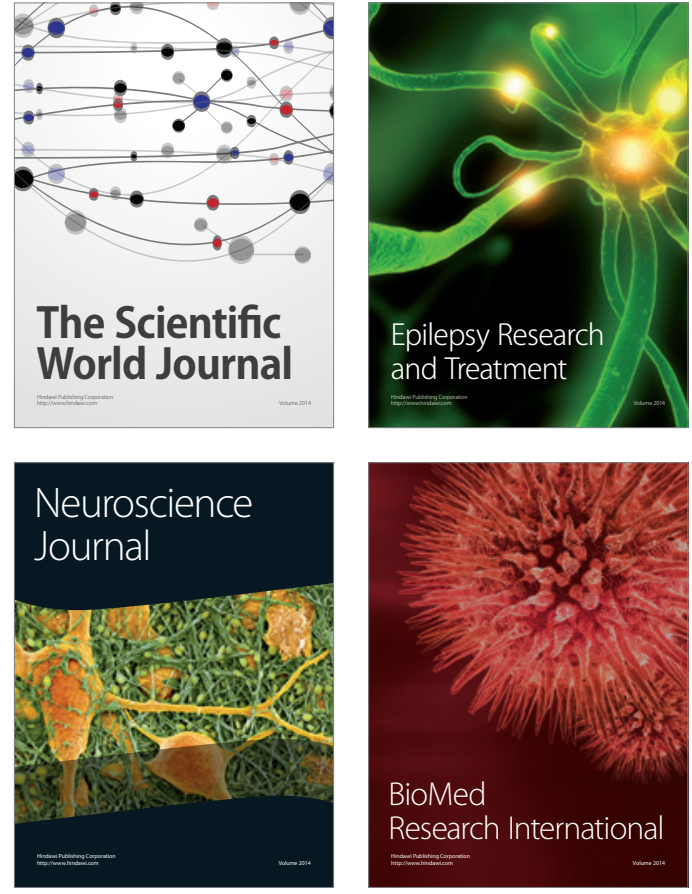

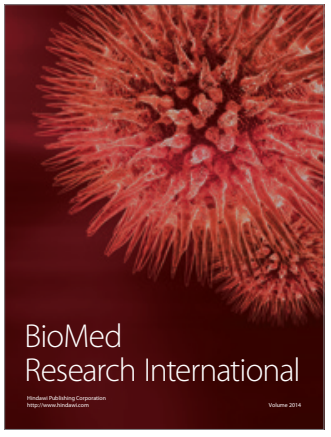

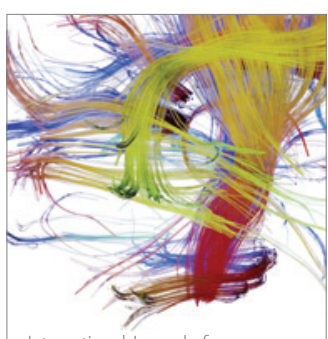

Brain Science

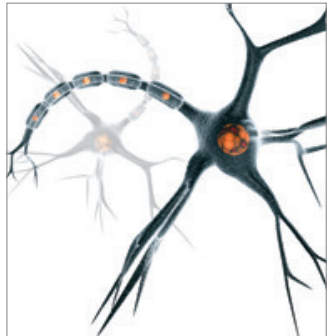

Neural Plasticity
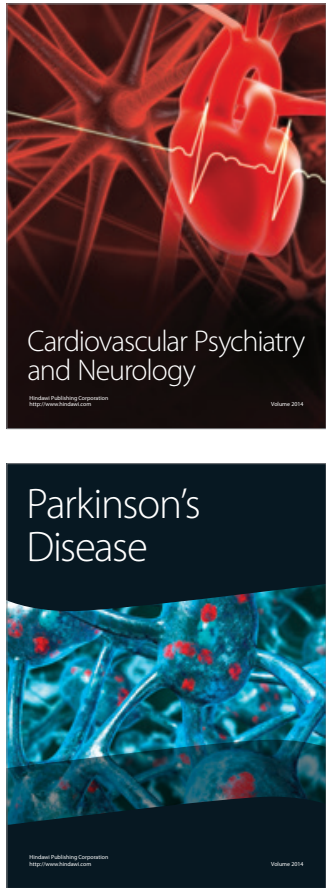